\section{The unbegun Big Bang}

SIR-While it is most plausible that "the Big Bang is an over-simple view of how the Universe began", as recently stressed by John Maddox", it need not be as "philosophically unacceptable" as he contends. My purpose is to dissent from the common but mistaken idea that Big Bang cosmologies imply "that there was an instant at which time literally began and, so, by extension, an instant before which there was no time".

In truth, the so-called 'initial' instant $t_{0}$ corresponds to a singularity of the spacetime model (as expressed by the Robertson-Walker metric) which has to be taken at face value: because the model is not defined for $t=t_{0}$, this value does not belong to its physical time domain. In other words, the continuity, or, more to the point, the contiguity of time breaks down. The range of physical time consists only of the open interval $] t_{0}, \infty[$; the initial time $t_{0}$ is not a moment in the life of the Universe, and does not belong to its past - which a fortiori cannot extend before $t_{0}$. As such, this out-of-reach instant may be said to be infinitely remote, irrespective of its finite numerical value on a conventional timescale. This crucial point has been stressed by Misner ${ }^{2}$ who proposes to treat it as "an essential element of cosmological theory" and suggests that we refine our concepts of time accordingly. His plea, however, has been little heard.

This situation is not so new: it is similar to the concepts of limit velocity $c$, or absolute zero for temperatures $T_{0}$, both of which mark true singularities in the relevant theoretical formalisms and can be considered as infinite, that is unreachable, values. Just as we have become accustomed to the apparent paradox of a zero temperature, below which the concept of temperature breaks down, so we should accept the idea of a time origin before which the concept of time makes no sense.

Furthermore, according to the theoretical framework of modern cosmology, namely general relativity, the choice of coordinates used to describe the Universe is arbitrary. Formally, the spatio-temporal parameters through which the RobertsonWalker metric is expressed, may be modified at will. The usual choice of cosmic time, although convenient, is by no means unique. Thus it is possible, through a suitable redefinition of time, to send back the birth of the Universe to (minus) infinity where, or rather when it seems to belong. Such a redefinition, already outlined by Misner ${ }^{2}$, can be derived through a simple group-theoretical analysis designed to restore the additivity of time, a property lost by the conventional cosmic time ${ }^{3}$. This new, linear time is defined by using the Universe itself as its own clock through its expansion, and leads to a suggestive re- expression of the Robertson-Walker metric. Naturally, on the linear scale, the lifespan of the Universe is infinite, so that never did the Big Bang begin. Thus, one may agree with the title of the article by John Maddox, Down with the Big Bang, provided it is understood as 'down in time' (and infinitely so).

Note that no new physics is involved in the introduction and use of linear time rather than cosmic time. Although it might be fruitful for some computational purposes, its main interest is conceptual.

There is certainly a great amount of work to be done for improving or even replacing the standard Big Bang theory as a description of the primitive stages of the

\section{Frozen Neanderthals}

SIR-Jared Diamond describes in his News and Views article ${ }^{1}$, "The recent spectacular discovery of the first reptile to survive natural freezing". He refers to Storey's 1988 report $^{2}$ that hatchlings of the Canadian painted turtle in the laboratory can survive the freezing of 52-53 per cent of total body water at $-4{ }^{\circ} \mathrm{C}$ and to comparable findings for certain frogs facts of great significance to their survival in nature. Diamond concludes that " $\mathrm{Al}$ though the discoveries of the first freezetolerant vertebrates seem to bring the dream of thawing out [and resuscitating a frozen Neanderthal] tantalizingly closer they actually push it even further into the realm of science fiction. Only by a series of specialized adaptations lacking in most animals do the painted turtle and the four frog species confine freezing to the extracellular space and so avoid lethal damage".

I agree that obtaining viable frozenthawed Neanderthals is science fiction not because they lack specialized adaptations, but rather because to survive millennia, they would have to be maintained at temperatures at which biochemical activity ceases, that is, below $-120^{\circ} \mathrm{C}$. Unfortunately, the very act of cooling a whole animal to such temperatures will increase the solute concentrations in its tissues 30 -fold or more over normal and will convert essentially all its body water to ice. The result, at least for vertebrates, will be instant death.

The first report of a vertebrate surviving partial freezing to -4 or $-5^{\circ} \mathrm{C}$ was in 1956, and concerned not a reptile or frog with specialized adaptations but a mammal with no adaptations other than the ability to survive cooling in the absence of freezing to $0^{\circ} \mathrm{C}$ and slightly below. In that year Smith and Lovelock ${ }^{3,4}$
Universe. Let us not confuse, however, the form and the meaning of our theories. If the present ones are certainly open to much scientific questioning, their philosophical status does not seem that disquieting - provided that our own understanding raises to match their subtleties. If, as John Maddox foresees, "creationists will have to retreat to the Big Bang", we have not too much to fear, as this retreat will be but an endless one.

\section{Physique Théorique,}

JEAN-MARC LÉVY-LeBLoND

Université de Nice,

Parc Valrose,

06034 Nice Cedex,

France

1. Maddox, J. Nature 340, 425 (1989)

2. Misner, C.W. Phys. Rev. 186, 1328 (1969)

3. Lévy-Leblond, J.-M. Am. J. Phys. (in the press).

reported that 30 per cent of golden hamsters survived, without apparent injury, the conversion to ice of $40-50$ per cent of their body water as the result of $40-60 \mathrm{~min}$ in liquid baths at $-5^{\circ} \mathrm{C}$. This result is not dissimilar from those for the painted turtle, except that all four of the latter exhibited no evidence of injury after a $12 \mathrm{~h}$ exposure in the partly frozen state to air at $-4{ }^{\circ} \mathrm{C}$, at which point half the body water was frozen.

Diamond is correct in emphasizing that survival of whole animals (or of most individual cells) after freezing requires that ice be confined to the extracellular space, but this does not demand that the animal possess special adaptations. Rather, it requires: that the first ice forms with little supercooling, and that it first appears in the extracellular space (which is usually the case); that the subsequent cooling rate is low enough to allow sufficient unfrozen cell water to leave the cells osmotically to keep the chemical potential of the residual cell water close to that of the external ice; or that the temperature does not drop to a level at which ice nucleation of the water in supercooled cells can occur ${ }^{5}$. Specialized adaptations do become important in enhancing the ability of cells and organisms to survive greater quantities of extracellular ice (either by the synthesis of cryoprotectants or by plasma-membrane alterations) or by enhancing their ability to supercool.

\section{Biology Division,}

\section{Oak Ridge National Laboratory,}

P.O. Box 2009 ,

Oak Ridge, Tennessee 37831-8077, USA

1. Diamond, J.M. Nature 339, 509-510 (1989).

2. Storey, K.B. et al. Proc. natn. Acad. Sci. U.S.A. 85, 8350 8354 (1988)

3. Smith, A.U. Proc. R. Soc. B 145, 407-426 (1956).

4. Lovelock, J.E. \& Smith, A.U. Proc. R. Soc. B145, 427-442 (1956).

5. Mazur, P. Am. J. Physiol. 247, C125-C142 (1984). 